\title{
Adaptively Induced Fluctuations for Multiuser Diversity: Two-dimensional Parameters and Cellular Interference
}

\author{
Rahul Dangui \\ Corporate R\&D \\ Qualcomm, Inc. \\ 5775, Morehouse Drive \\ San Diego, California \\ Email: dangui@comm.csl.uiuc.edu
}

\author{
Bruce Hajek \\ Dept. of Electrical and Computer Engineering and \\ the Coordinated Science Laboratory \\ University of Illinois at Urbana-Champaign \\ Urbana, Illinois \\ Email: b-hajek@comm.csl.uiuc.edu
}

\begin{abstract}
This study extends recent work on using adaptive phase fluctuations to enhance multiuser diversity in the downlink of a cellular network. Feedback at the base station is limited and Rayleigh fading is assumed.

Our main findings are presented along three main axes.

First, we study a single-cell environment with adaptive fluctuations induced between multiple transmit antennae at the base station. Phase fluctuations of three transmit antennae are induced based on a two-dimensional mapping of their relative phases. A one to two dB improvement of the performance is obtained over the two-antennae case. For two transmit antennae, we also investigate another two-dimensional problem by jointly varying the phase difference and power allocation between two antennae. Our simulations show somewhat surprisingly that an equal power-splitting with adaptive phase fluctuations provides better performance than the joint strategy.

Second, we take into account the effects of intercell interference. The impact of neighboring cells using the same transmission frequency and executing the same adaptive algorithm is accounted for. A simple model is studied by considering a single interfering cell with parameters chosen to account for the power of the six nearest interfering cells. Our simulations and analysis probe the consequences of the fact that intercell interference noise is burstier than thermal noise. We find that multiuser diversity is more effective against interference than against Gaussian thermal noise with equal power. However, the interfering noise tends to become Gaussian when the number of interferers increases. The performance is also observed to be insensitive to the phase variation strategy of the interfering station. We further consider a more detailed model involving the simultaneous simulation of the six nearest interfering cells, with users randomly distributed in the cells.
\end{abstract}

Third, fairness issues are taken into account. We observe that fairness decreases throughput but we still observe a multiuser diversity gain.

\footnotetext{
${ }^{1}$ Keywords - Wireless communications; Rayleigh fading channels; multiuser diversity; multiple antennae; propagation; intercell interference; power control; Monte Carlo simulations; fairness mechanisms; scheduling algorithms;

${ }^{1}$ This work was supported in part by the National Science Foundation under grants NSF CCR 99-79381 and NSF ITR 00-85929. A shorter version of this paper was presented at the IEEE Vehicular Technology Conference, Dallas, Fall 2005. This work is based on the M.S. thesis of the first author, completed at UIUC.
}

\section{INTRODUCTION}

The concept of multiuser diversity is central to this paper and may be described as follows. It is reasonable to assume that the channels between distinct users and a base station fade independently. If the base station has information about the channel quality, it can take advantage of this independence to transmit in a given time slot only to the user with the best channel. This is the multiuser diversity effect.

This concept has its roots in information theoretic results as follows. First, Knopp and Humblet [1] showed that in the case of a single-cell uplink with multiple users communicating over time-varying channels, an optimal strategy from a capacity point of view is to receive data at any given time only from the user with the best channel. Assuming that the channels can be perfectly tracked at the mobile users and the base station, Tse [2] showed that the total downlink information capacity can be achieved by transmitting only to the user with the best channel at any time. Viswanath et al. [3] proposed to further improve the performances of [2] by inducing fluctuations through the phase variation of an additional transmit antenna at the base station. They studied this phenomenon in the case of complete feedback: this is called opportunistic beamforming. The same signal is transmitted though both antennae with the phase difference being varied randomly. The use of multiple antennae enables large and fast channel fluctuations, even in slow fading or little scattering environments. Moreover, this requires no more signal processing than the one-antenna case. It is therefore easier to implement than techniques based on space-time processing or smart antennae.

In this paper, we extend the work of [4] by considering a two-dimensional search which can be applied to phase fluctuations of three transmit antennae or joint power-phase fluctuations of two transmit antennae. We also address the issues of intercell interference and fairness. 


\section{Multiple transmit Antennae with AdAptive PHASE FLUCTUATIONS IN ONE CELL}

\section{A. Brief description}

The system is composed of a central base station with $N$ transmit antennae and $K$ mobile users. The same signal with different phases is transmitted by the transmit antennae. The presence of more than one antenna at the base station introduces additional paths between the transmit antennae and the considered mobile user. This results in changes in the interference pattern, i.e., the combination of the signal paths can either interfere constructively or destructively. By controlling the phases, we can thus create constructive interference, resulting in a higher Signal to Noise Ratio (SNR).

\section{B. System model}

The base station and the mobile users communicate over time-varying fading channels with additive white Gaussian noise (AWGN) channels. Time slots are indexed by $l$. The channel coefficients are treated as constant during a time slot of fixed length $l$. The coefficient $h_{i k}$ is the channel gain between the user $k(1 \leq k \leq K)$ and the transmit antenna $i(1 \leq i \leq N)$. The random variables $\left(h_{i k}\right)$ are assumed to be mutually independent and circular symmetric complex Gaussian with mean 0 and variance $2 \sigma^{2}$.

The transmit antenna $i$ is allocated a fraction $\alpha_{i}^{2}$ of the total transmit power, which is assumed constant, and transmits in slot $l$ the signal $x(l) \alpha_{i}(l) e^{j \theta_{i}(l)}$, where the phases $\left(\theta_{i}\right)_{1<i<N}$ are being varied as per our strategy further described. We assume Rayleigh fading commonly used for mobile users in urban areas, as well as Gauss-Markov time evolution.

\section{Service model}

Each time slot consists of alternate test and service periods. First, the mobile user $k$ records its received SNR as per:

$$
S N R_{k}(l)=\left|\sum_{i=1}^{N} h_{i k}(l) \alpha_{i}(l) e^{j \theta_{i}^{\prime}(l)}\right|^{2}
$$

where $\theta_{i}^{\prime}$ is the relative phase between antenna $i$ and antenna 1, i.e., $\theta_{i}^{\prime}=\theta_{i}-\theta_{1}, 1 \leq i \leq N$ and feeds it back to the base station, which then serves the strongest SNR user.

\section{Case of three antennae at the base station}

The scheme used for controlling the phase differences $\theta_{2}^{\prime}$, $\theta_{3}^{\prime}$ in our study is given by the following update equations:

$$
\begin{gathered}
\theta_{2}^{\prime}(l+1)=\theta_{2}^{\prime}(l)+\phi \Delta \theta(l) \bmod .2 \pi \\
\theta_{3}^{\prime}(l+1)=\theta_{3}^{\prime}(l)+\Delta \theta(l) \bmod .2 \pi
\end{gathered}
$$

where $\phi=\frac{1+\sqrt{5}}{2}$ and

$$
\Delta \theta(l)=B \exp \left(-\gamma S N R^{*}(l)\right)
$$

Here $S N R^{*}(l)=\max _{1 \leq k \leq K} S N R_{k}(l)$, and $B$ and $\gamma$ are parameters of the system which we determine by an off line joint-search. Given the knowledge of the channel coefficients, there exist optimal values of $\theta_{2}^{\prime}$ and $\theta_{3}^{\prime}$ maximizing the throughput and corresponding to the beamforming situation.

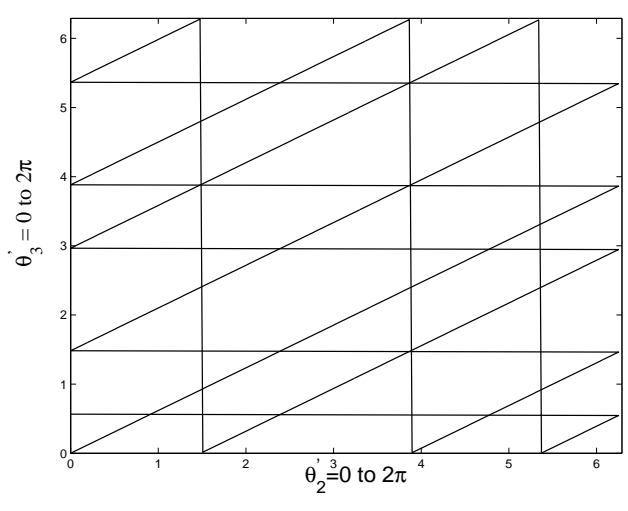

Fig. 1. Golden-ratio mapping.

They are both functions of the channel coefficients and are consequently time slot dependent as the channels are timevarying.

By inducing fluctuations, our goal is for the phases to spend most of the time around the good regions of the joint-space where $S N R_{\theta_{2}^{\prime}, \theta_{3}^{\prime}}^{*}(l)$ is high so that we can ensure a good average throughput on the long run. As the base station is only aware of the $S N R$ and not of the individual channel coefficients, it does not know the optimal phase values but needs them to move around the complete square $[0,2 \pi]^{2}$ to track them efficiently. A larger $S N R^{*}(l)$ results in $\left(\theta_{2}^{\prime}(l)\right.$; $\left.\theta_{3}^{\prime}(l)\right)$ dwelling longer in the good regions of the phase space: the trajectory slows down and the transmission takes place at a higher rate. A large value of $\gamma$ is only good in the vicinity of the optimum. Therefore, a careful choice of $B$ linked to the choice of $\gamma$ can somewhat mitigate the negative effect in bad regions of the phase.

In the case of two antennae with equal power split, the search for a good phase $\theta_{2}^{\prime}$ is one-dimensional. Since we are considering three antennae with equal power split, a twodimensional sampling technique is needed. To avoid large phase jumps, (3) uses a one-dimensional trajectory technique which rapidly fills out the two-dimensional phase space. The sampling technique used which we call golden-ratio mapping enables us to go through all the possible values of $\theta_{3}^{\prime}$ when $\theta_{2}^{\prime}$ circles repeatedly through $[0 ; 2 \pi]$. The dense and uniform coverage of $[0 ; 2 \pi] \times[0 ; 2 \pi]$ stems from the irrationality of $\phi$. This is illustrated in Fig. 1. The plot has been done for $\theta_{2}^{\prime}$ circling 5 times around the interval $[0 ; 2 \pi]$.

\section{E. Simulations and observations}

We present simulations for Rayleigh fading and low SNR regime and two values of the Gauss-Markov parameter $a$. We take the throughput to be proportional to SNR, which is reasonable if the network is operating in a low SNR regime. A larger value of $a$ corresponds to a faster time variation of the channel. In the simulations, the optimal values of the parameters B and $\gamma$ are found by an off line search through the joint space of $(B, \gamma)$, such that the average throughput per time slot is maximized using these parameters. 


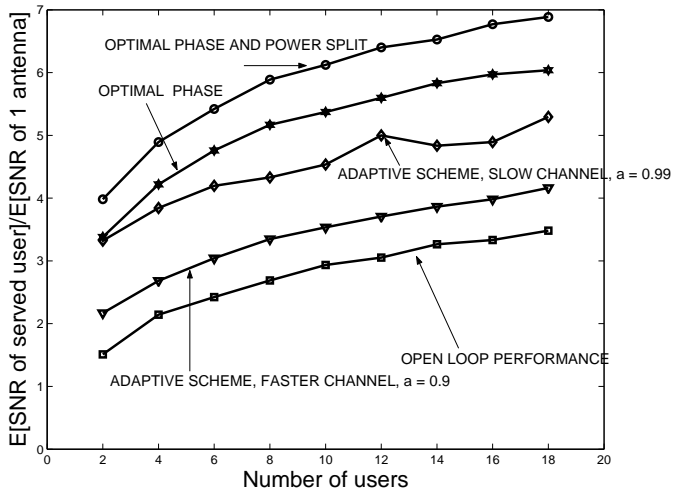

Fig. 2. Adaptive scheme for Rayleigh fading in the low SNR regime, case of 3 antennae.

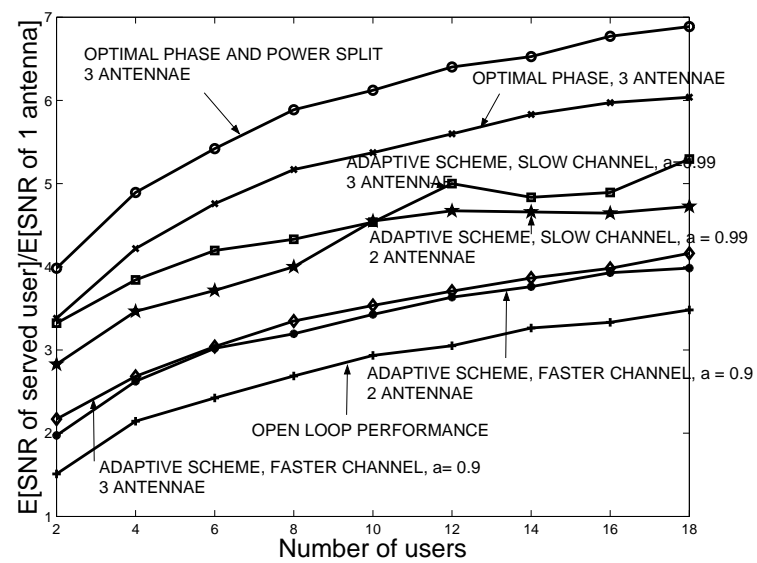

Fig. 3. Performance of the adaptive scheme for Rayleigh fading in the low SNR regime, comparison 2 antennae/3 antennae.

Fig. 2 illustrates the performance for three transmit antennae. We observe that the adaptive scheme offers a good improvement of the performance in comparison to the openloop performance where $\theta_{2}^{\prime}$ varies randomly without any use of the feedback. The gain is around $3 \mathrm{~dB}$ in the case of a slow channel $(a=0.9)$ and $1 \mathrm{~dB}$ in the case of a faster channel ( $a=0.99$ ), uniformly over the number of users from 2 to 20. It is also noticeable that beamforming, where we have optimal phase and power splitting or optimal phase and equal power splitting, offers better results: 1 to $2 \mathrm{~dB}$ more, which is logical. However this requires a perfect knowledge of the channels, which is typically unavailable. We also observe that a slow channel where $a$ has the value 0.99 , for example corresponds to a higher throughput than a faster channel where $a$ equals 0.9. This is due to the adaptive scheme having enough time to adapt itself to the values of the channel, and thus to find a better value for the control parameters. Moreover, this shows that even a small decrease in the value of $a$ can provide a good improvement of the performance. Fig. 3 compares the performance for two transmit antennae (one-dimensional search) and three transmit antennae (two-dimensional search). We see that for a fast channel corresponding to $\mathrm{a}=0.9$, the performances are not really improved: the gain is only around
$0.1 \mathrm{~dB}$. However, when the evolution in time of the channels is slower, for example $a=0.99$, the improvement is nearly $1 \mathrm{~dB}$. Hence in this case, having three antennae at the base station offers a slight improvement in the performance. This improvement increases as the channel gets slower.

F. Further investigations and simulations in the case of two antennae at the base station: joint time-varying power-phase allocations

The study is done in the case of two antennae at the base station. We investigate now the effect of a joint variation in time of the power allocation and the phase fluctuations on the performance of the system. This issue is of interest because a joint variation of the power splitting and the phases may possibly improve the performance compared to an equal power splitting. Indeed, some upper-bounds on the performance in the case of joint control of the phase and power among the transmit antennae are presented in [4]. The optimal performance corresponding to the upper-bound is obtained by beamforming to the best user and is based on the CauchySchwarz inequality.

In the previous subsection, we introduced a two-dimensional search through the phase space in order to track the optimum phase configuration. The power was allocated equally among the three antennae : $\alpha_{1}^{2}=\alpha_{2}^{2}=\alpha_{3}^{2}=\frac{1}{3}$. For two antennae, there is only one phase difference to consider. We therefore consider introducing a second dimension corresponding to a time-varying power allocation, using a scheme similar to the one presented earlier and based on the golden ratio. Our approach is based on the fact that as in the previous section, we will look for a two-dimensional search tracking the optimal configuration and spending as much time as possible in its vicinity. The scheme will be based on the golden-ratio mapping introduced previously with some slight modifications due to $\alpha_{1}^{2}$ being defined over $[0,1]$.

For two antennae at the base station, the phases and the power allocation corresponding to beamforming to the user $k$ are given by (the time slot $l$ is omitted for clarity purposes)

$$
\begin{aligned}
& \alpha_{1}^{2}=\frac{\left|h_{1 k}\right|^{2}}{\left|h_{1 k}\right|^{2}+\left|h_{2 k}\right|^{2}} \\
& \alpha_{2}^{2}=\frac{\left|h_{2 k}\right|^{2}}{\left|h_{1 k}\right|^{2}+\left|h_{2 k}\right|^{2}}
\end{aligned}
$$

and

$$
\begin{gathered}
\theta_{1}=-\arg \left(h_{1 k}\right) \\
\theta_{2}=-\arg \left(h_{2 k}\right)
\end{gathered}
$$

where $h_{i k}$ is $\mathbf{C N}\left(0,2 \sigma^{2}\right)$.

The computation of the $S N R$ requires only the knowledge of $\alpha_{1}^{2}$ and $\theta_{2}^{\prime}=\theta_{2}-\theta_{1}$. We can show that under the assumption on the distribution of $h_{i k}, \alpha_{1}^{2}$ is uniformly distributed over $[0 ; 1]$ (see Appendix I for some explanations) and thus has mean $\frac{1}{2}$. As previously, our mapping must be able to efficiently track the optimum in the two-dimensional space. We therefore use a mapping between $\theta_{2}^{\prime}$ and $\alpha_{1}^{2}$ such that $\alpha_{1}^{2}$ spends 


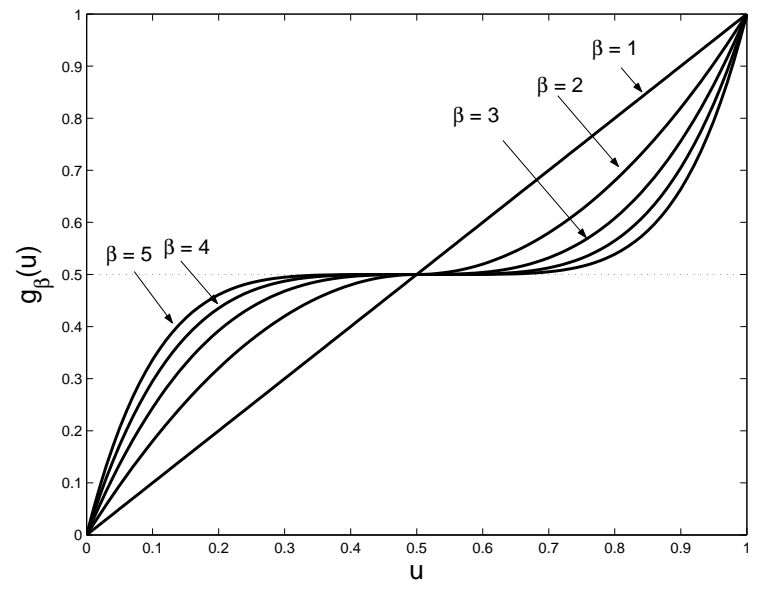

Fig. 4. Plot of the parametric function $g_{\beta}(u)$ over $0 \leq u \leq 1$ for $\beta=$ $1,2,3,4,5$.

time around $\frac{1}{2}$ which represents the situation of equal power splitting, namely

$$
\alpha_{1}^{2}=g_{\beta}(u)
$$

where $\beta$ is a real number $(\beta \geq 1)$ and $g_{\beta}(u)$ is defined as

$$
g_{\beta}(u)=\frac{1}{2}\left(1+(2 u-1)|2 u-1|^{\beta-1}\right) .
$$

A larger $\beta$ biases $\alpha_{1}^{2}$ closer to $\frac{1}{2}$ as in illustrated in Fig. 4 . The parameter $\beta$ is chosen so that we obtain the best performances.

The variable $u$ is mapped through a golden-ratio mapping to $\theta_{1}^{\prime}$ :

$$
\begin{aligned}
\theta_{1}^{\prime}(l+1) & =\theta_{1}^{\prime}(l)+\Delta \theta(l) \\
u(l+1) & =u(l)+\phi \Delta \theta(l)
\end{aligned}
$$

where

$$
\Delta \theta(l)=B \exp \left(-\gamma S N R^{*}(l)\right)
$$

Fig. 5 illustrates this situation for a low $S N R$ regime in the case of a Rayleigh fading and a slow channel where $a=0.99$. The mapping is described by the value of the parameter $\beta$ which is taken to be equal to 2 or 3 . It is a measure of the speed of the trajectory around $\alpha_{1}^{2}=\frac{1}{2}$.

Typically, the curve for $\beta=2$ spends more time around it, so it should give better results than $\beta=3$. We observe that a time-varying golden-ratio power splitting mapping does not improve the performance of the scheme on the long term; on the contrary, it degrades performance. Thus, it seems that an equal power allocation among the antennae offers a better performance.

\section{MODELING THE INTERFERENCE OF NEIGHBORING CELLS}

\section{A. Description of the model, key assumptions}

As seen in the previous section, no intracell interference has to be considered as only one user is served per time slot in a given cell. However, the previous downlink scheme may be implemented in each of many cells. Therefore, other cells

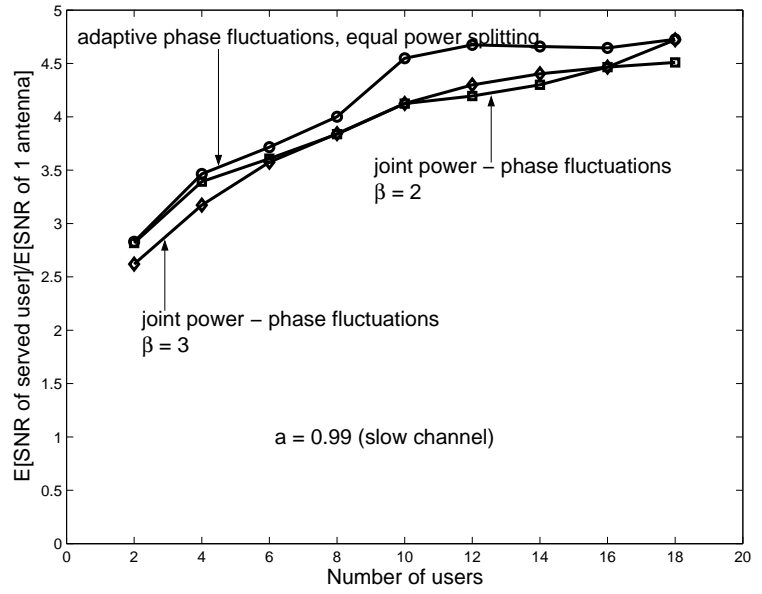

Fig. 5. Performance of the adaptive phase-power splitting scheme in a low SNR regime in the case of Rayleigh fading (2 antennae).

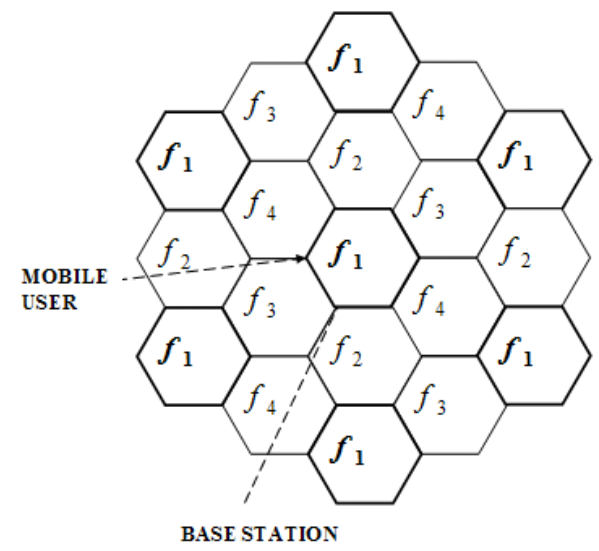

Fig. 6. Cells with a frequency reuse of $\frac{1}{4}$.

interact with the one considered, affecting the performance. In this section, we address the issue of intercell interference. The model is a combination of probability distributions and of a geometric point of view through a path-loss model. Cells are modeled as hexagons of radius $R$ and the studied cell is denoted cell 0 . Our approach is based on [5] and illustrated in Fig. 6. We consider a frequency reuse factor equal to $\frac{1}{4}$. The following key additional assumptions are made:

- Each base station has two antennae and is located at the center of its cell.

- During the time slot where a given mobile user in cell 0 communicates with the base station of its cell, the interfering base stations transmit at the same time, i.e., we have a synchronization of the time slots.

- The path-loss follows the empirical model $P_{r}(d) \propto$ $(d)^{-4}$ where $P_{r}$ is the average received signal strength at a distance $d$ of the transmitter. 
This last formula can for example be found in [5]. It shows that the average received signal strength $P_{r}$ at any point at distance $d$ decays as a power law of the distance of separation $d$ between the receiver and a transmitter. The path-loss exponent is chosen equal to 4 because we consider a crowded urban environment.

The interference is generated primarily by the six nearest interfering cells using the same frequency (corresponding to the first layer of interfering cells). These cells are denoted cell $1, \ldots$, cell 6 .

For each user $k$ of cell 0 , the interfering signal from cell $j$ $(1 \leq j \leq 6)$ is simply taken to be a signal of the same nature as the desired signal from the base station in the same cell but with an attenuation factor $a_{k}^{(0 j)}$ chosen according to the path-loss previously mentioned, i.e., $a_{k}^{(0 j)}=\left(d_{k}^{(0 j)} / d_{k}^{(0)}\right)^{4}$, where for $1 \leq i \neq j \leq 6, d_{k}^{(i j)}$ is the distance from a user $k$ in cell $i$ to the base station of cell $j$ and $d_{k}^{(i)}$ is the distance from a user $k$ in cell $i$ to its own base station.

Extending the notations of the previous section to the context of six interfering cells, we adopt the following notations for $1 \leq i \neq j \leq 6$ :

- $\left|x^{(i)}(l)\right|^{2}$ is the total transmit power of the base station of cell $i$ in time-slot $l$.

- $\alpha_{1}^{(i)}(l)$ and $\alpha_{2}^{(i)}(l)$ represent the allocation of power among the two transmit antennae of the base station of cell $i$ in time-slot $l: \alpha_{1}^{(i)^{2}}+\alpha_{2}^{(i)^{2}}=1$.

- $h_{1 k}^{(i)}(l)$ and $h_{2 k}^{(i)}(l)$ represent the channel coefficients between the user $k$ in cell $i$ and the transmit antenna 1 (resp. 2) of the base station of cell $i$ in time-slot $l$.

- $\widetilde{h}_{1 k}^{(i j)}(l)$ and $\widetilde{h}_{2 k}^{(i j)}(l)$ represent the interfering channel coefficients between the user $k$ of cell $i$ and the transmit antenna 1 (respectively 2) of the base station of cell $j$ in time-slot $l$.

- $\theta^{(i)}(l)$ is the phase difference between the two transmit antennae of the base station of cell $i$ in time-slot $l$.

A random variable $U_{k}^{(0)}$ refers to the desired signal for a user $k$ in cell 0 . It is defined as follows for two transmit antennae in cell 0 :

$$
U_{k}^{(0)}(l)=h_{1 k}^{(0)}(l) \alpha_{1}^{(0)}(l)+h_{2 k}^{(0)}(l) \alpha_{2}^{(0)}(l) e^{j \theta^{(0)}(l)}
$$

Each random variable $\widetilde{U}_{k}^{(0 j)}(l)$ refers to the interfering signal from the transmit array in cell $j$ to the user $k$ in cell 0 and is defined as follows for two transmit antennae and adaptive phase fluctuations in cell $j$ :

$$
\widetilde{U}_{k}^{(0 j)}(l)=\widetilde{h}_{1 k}^{(0 j)}(l) \alpha_{1}^{(j)}(l)+\widetilde{h}_{2 k}^{(0 j)}(l) \alpha_{2}^{(j)}(l) e^{j \theta^{(j)}}(l)
$$

We assume the base stations have the same total power; hence, we suppose that for $1 \leq i, j \leq 6$ : $\left|x^{(i)}\right|^{2}=\left|x^{(j)}\right|^{2}=P$ where $P$ is a constant. The $S I N R$ in the time slot $l$ for the served mobile user $k$ in cell 0 (for two antennae at each base station and given the channel gains) is thus given by

$$
\operatorname{SINR}_{k}^{(0)}(l)=\frac{\left|U_{k}^{(0)}(l)\right|^{2}}{\sum_{j=1}^{j=6} a_{k}^{(0 j)}(l)\left|\widetilde{U}_{k}^{(0 j)}(l)\right|^{2}+P_{\text {thermalnoise }} / P}
$$

A value for the ratio $\frac{P_{\text {thermalnoise }}}{P}$ is determined as follows: We take a reference $S N R$, denoted by $S N R_{\text {ref }}$ computed when no intercell interference is considered, and there is only one antenna at the base station. Namely, $10 \log \left(\frac{E\left[\left|h_{1 k}^{(0)}(l)\right|^{2}\right]}{P_{\text {thermalnoise }} / P}\right)=S N R_{\text {ref }}$.

Since $\left|h_{1 k}^{(0)}(l)\right|^{2}$ is exponentially distributed with parameter $\frac{1}{2 \sigma^{2}}$, this brings, assuming a nominal value $S N R_{\text {ref }}=5 \mathrm{~dB}$, $\frac{P_{\text {thermalnoise }}}{P}=\frac{2 \sigma^{2}}{\sqrt{10}}$ as the thermal noise is assumed to be Gaussian. We have finally our simplified model:

$$
\operatorname{SINR}_{k}^{(0)}(l)=\frac{\left|U_{k}^{(0)}(l)\right|^{2}}{\sum_{j=1}^{j=6} a_{k}^{(0 j)}(l)\left|\widetilde{U}_{k}^{(0 j)}(l)\right|^{2}+\frac{2 \sigma^{2}}{\sqrt{10}}}
$$

This expression has been obtained for two antennae per base station. A similar expression for any number of transmit antennae at the base station in each cell can be easily obtained.

\section{B. Fairness, delay of the scheme}

In our context, the resource is the amount of data and the competing users are the mobile stations in a given cell. In a multiuser diversity scheme, only one user gets all the throughput in a given time slot. The chosen one is the one with the best $S I N R$. This approach is beneficial to the throughput [1], but does not ensure fairness. Namely, in our scheme, a small number of users with the best channels might receive most of the throughput, whereas the others will be deprived of data. The approach is not relevant in the context of delaylimited data where we want the mobile users to receive their respective datas in a reasonable amount of time.

A generalized definition of fairness has been introduced in [6] by Mo and Walrand. It presents a family of utility functions, parameterized by a nonnegative parameter $\alpha$ varying between 0 and $+\infty$. A large value of $\alpha$ promotes equality of throughputs and $\alpha$ near zero promotes maximizing the sum of throughputs. The family of functions $\left(U_{\alpha}(x)\right)_{\alpha \geq 0}$ is defined for a given $\alpha$ by [6]:

- If $\alpha=1: U_{\alpha}(x)=\log (x)$

- Otherwise, $U_{\alpha}(x)=\frac{x^{1-\alpha}}{1-\alpha}$

In our study, for a given cell $i$ and a given time-slot $l$, our fairness approach will be based on $\left(U_{\alpha}\left(T_{k}^{(i)}(l)\right)\right)_{1 \leq k \leq K_{i}}$, where $T_{k}^{(i)}(l)$ is the average cumulative throughput through user $k$ of cell $i$, until time slot $l$, defined as follows:

$$
T_{k}^{(i)}(l)=\frac{1}{l} \sum_{p<l} f\left(\operatorname{SIN} R_{k}^{(i)}(p)\right)
$$


where the sum is limited to the slots $p$ such that $k^{*(i)}(p)=k$, i.e., to the slots where $k$ is the chosen user in cell $i$ (as per our strategy described below).

- In the low-SNR regime, $f(x)=x$.

- In the high-SNR regime, $f(x)=\log (1+x)$.

The user $k$ selected for downlink in cell $i$ slot $l$ is $k^{*(i)}(l)$ :

$$
k^{*(i)}(l)=\arg \max _{1 \leq k \leq K_{i}} f\left(S I N R_{k}^{(i)}(l)\right) U_{\alpha}^{\prime}\left(T_{k}^{(i)}(l)\right) .
$$

The choice (19) is the one maximizing the aggregate utility, $\sum_{k=1}^{K_{i}} U_{\alpha}\left(T_{k}^{(i)}(l+1)\right)$, as predicted by the first order increase based on Taylor's theorem. The choice (19) can be written using the definition of $U_{\alpha}(x)$ as:

$$
k^{*(i)}(l)=\arg \max _{1 \leq k \leq K_{i}} \frac{f\left(S I N R_{k}^{(i)}(l)\right)}{\left[T_{k}^{(i)}(l)\right]^{\alpha}}
$$

Consequently, the selection of the mobile user $k^{*(i)}(l)$ is based not only on the instantaneous quality of its channel, but also on the amount of throughput it has been able to receive. Similarly, we slightly modify (4) so the phase difference spends most of the time in good regions of the phase space, which correspond now to high values of $\sigma^{*(i)}(l)=\frac{f\left(\operatorname{SIN}_{k^{*(i)}(l)}^{(i)}(l)\right)}{\left[T_{k^{*(i)}(l)}^{(i)}(l)\right]^{\alpha}}$. Therefore, the phase jump in cell $i$ and in slot $l$ is determined as follows:

$$
\Delta \theta^{i}(l)=B \exp \left(-\gamma \sigma^{*(i)}(l)\right) .
$$

The steps are therefore the following:

- The $S I N R$ of a mobile user $k$ is computed based on (17).

- The chosen user $k^{*(i)}(l)$ in time slot $l$ and in cell $i$ is chosen based on (20) to account for fairness.

- The phase fluctuations in each of the seven cells are based on (21).

Note that the value $\alpha=0$ in the above fairness scheme corresponds to the approach (taken in the previous subsection), where the only concern is maximizing the throughput. Choosing $\alpha=1$ corresponds to proportional fairness [7], whereas choosing $\alpha \rightarrow+\infty$ corresponds to a max-min fairness approach.

\section{INTERFERENCE FROM A Single Fictitious CELL}

Limiting our network to only two cells is part of an attempt to capture the main features of inducing adaptive phase fluctuations at the network level through a basic, lowcomplexity model. The main simplification stems from the fact the two-cell system is self-sufficient, in that each of the two cells is the only interfering cell affecting the other one. Hence, studying the adaptive phase strategy in each cell does not require any information other than the interfering channels and the value of the phase in the other cell. Considering the phase-adaptive algorithm running simultaneously in six interfering cells presents some computational complexity which we discuss in the next section. By considering only one cell instead of the six nearest cells, we expect to simplify the model and overestimate the burstiness of the interference noise. We adapt the general notations introduced in the previous section to this simplified model as follows: the cell that we study is called cell 0 and the interfering cell (called single interferer or supercell) is represented by the index $S$.

We evaluate the performance of multiuser diversity with adaptive fluctuations for three strategies in the interfering cell:

- Case 1: only with the thermal noise due to the noisy channel between the transmit antenna and the receiver: we do not consider the impact of other cells.

- Case 2: with a mixture of the previously mentioned thermal noise and intercell interference requiring various levels of detail.

- Case 3: with a thermal noise which has the same total average power as the mixture.

The first case does not consider the dependence between the performances in distinct cells. A comparison between the performance in the second and third cases will determine whether the thermal or the intercell noise degrades the performance more at a given noise power.

\section{A. Choice of the parameters}

Within our simplified model and using the notations of last section, the $S I N R$ of a user $k$ in cell 0 and in time slot $l$ is given by

$$
\begin{aligned}
& S I N R_{k}^{(0)}(l)= \\
& \frac{\left|h_{1 k}^{(0)}(l) \alpha_{1}^{(0)}(l)+h_{2 k}^{(0)}(l) \alpha_{2}^{(0)}(l) e^{j \theta^{(0)}(l)}\right|^{2}}{A_{k}^{(0 S)}(l)\left|\widetilde{h}_{1 k}^{(0 S)}(l) \alpha_{1}^{(S)}(l)+\widetilde{h}_{2 k}^{(0 S)}(l) \alpha_{2}^{(S)}(l) e^{j \theta(S)}(l)\right|^{2}+\frac{2 \sigma^{2}}{\sqrt{10}}}
\end{aligned}
$$

where $A_{k}^{(0 S)}(l)$ is a coefficient which is chosen to account for the mean interference from the interfering cell to the $k^{t h}$ user in time slot $l$.

As we study a worst-case situation and in order to have a computationally tractable model, we decide to choose $A_{k}^{(0 S)}(l)$ without dependence on $k$ and $l$. We choose a geometric approach to obtain the interfering coefficients corresponding to the worst position in cell 0 . Neglecting the influence of the thermal noise, the signal-to-interference ratio $(S I R)$ can be computed through simple geometrical considerations:

- For a user on the middle of one side of cell $0, \frac{P_{\text {interf }}}{P_{\text {signal }}}=$ $\frac{1}{9^{2}}+\frac{2}{13^{2}}+\frac{2}{21^{2}}+\frac{1}{25^{2}}$, so the average signal-to-interference ratio $S I R$, defined by $10 \log \left(\frac{P_{\text {signal }}}{P_{\text {interf }}}\right) \mathrm{dB}$, is around 15 $\mathrm{dB}$.

- Similarly, for a user at a vertex, the average $S I R$ is approximately equal to $12.5 \mathrm{~dB}$.

The user at a vertex has a smaller $S I R$ than the user on the middle of one side and presents indeed the worst position on the border of the cell as far as the $S I R$ is concerned.

We set $A_{k}^{(0 S)}(l)=0.1$ for $1 \leq k \leq K_{0}$ in (22). This means that the interfering signal is attenuated on average $10 \mathrm{~dB}$ below the desired signal. We tend to overestimate the power of the noise obtained by the geometrical model because we study a worst-case situation. 


\section{B. Simulations and observations}

1) Single transmit antenna at the base station: Let $V_{k}^{(0)}=$ $\left|h_{1 k}^{(0)}(l)\right|^{2}$ and $\widetilde{V}_{k}^{(S)}=\left|\widetilde{h}_{1 k}^{(0 S)}(l)\right|^{2}$ represent respectively the power attenuation in the signals sent from the base station in cell 0 and in cell $S$. These random variables are mutually independent and exponentially distributed with parameter $\lambda=\frac{1}{2 \sigma^{2}}$. They are also independent across the users $k$ by assumption. The $S I N R$ for mobile user $k\left(1 \leq k \leq K_{0}\right)$ in cell 0 takes therefore the simplified form in the three cases mentionned above (the subscript 1 and the time slot $l$ are omitted for convenience):

Case 1:

$$
\left.S I N R_{k}^{(0)}=\frac{\lambda V_{k}^{(0)}}{\frac{1}{\sqrt{10}}} \text { (thermal noise only }\right)
$$

Case 2:

$S I N R_{k}^{(0)}=\frac{\lambda V_{k}^{(0)}}{\frac{1}{\sqrt{10}}+\frac{\lambda}{10} \widetilde{V}_{k}^{(S)}}$ (thermal noise and inter ference)

Case 3:

$S I N R_{k}^{(0)}=\frac{\lambda V_{k}^{(0)}}{\frac{1}{\sqrt{10}}+\frac{1}{10}} \quad$ (equivalent thermal noise)

The performance is given by $\max _{1 \leq k \leq K_{0}} S I N R_{k}^{(0)}(l)$, where $K_{0}$ is the total number of users in cell 0 . The average performance over an infinite time-horizon can be computed in the low SNR regime by $M=\operatorname{Emax}_{1 \leq k \leq K_{0}} S I N R_{k}^{(0)}$, where $S I N R_{k}^{(0)}$ takes one of the three above forms (23)(25) depending on the nature of the noise in each case.

We want to compare the performance in these three cases and a simple way to do it is to indicate the rate at which $M$ grows for large values of $K_{0}$. From a practical point of view, this approach only gives a rough idea of the performance because the number of users in a cell is limited to a small number, usually less than 20 .

Let $1 \leq i \leq 3$ specify the index of the case considered. It can be shown (see Appendix II) that:

- $M_{1} \sim R_{1} \triangleq \sqrt{10} \log K_{0}$

- $M_{2} \sim R_{2} \triangleq \sqrt{10} \log K_{0}$

- $M_{3} \sim R_{3} \triangleq \frac{\sqrt{10}}{1+\frac{1}{\sqrt{10}}} \log K_{0}$

where we write $M_{i} \sim R_{i}$ to mean $\frac{M_{i}}{R_{i}} \longrightarrow 1$ in probability as $K_{0} \longrightarrow \infty$, i.e., for any $\epsilon>0, P\left(\left|\frac{M_{i}}{R_{i}}-1\right|>\epsilon\right) \rightarrow 0$ as $K_{0} \rightarrow \infty$.

Note that $R_{1}=R_{2}$, so that the addition of intercell interference does not decrease the throughput at all, in an asymptotic sense. In the next section, this is born out in simulations for even moderate numbers of users. In comparison, $R_{3}$ is smaller than $R_{1}$ by $-10 \log _{10} \frac{1}{1+\sqrt{10}} \approx-1.2 d B$, so that if the intercell interference were replaced by white noise with the same power, there would be loss in throughput. Though the difference $1.2 \mathrm{~dB}$ is moderate, it could be larger in scenarios with stronger intercell interference.
2) Adaptive fluctuations for two antennae at the base station: We simulate adaptive fluctuations in both cells at the same time, the update of the phase in one cell affecting the update in the other cell through the value of the $S I N R$ and consequently, the choice of the served user. The performance is studied in cell 0 as a function of the numbers of receivers $K_{0}$ in cell 0 for many given values of $K_{S}$ (number of mobile users in cell $S$ ).

The simulations are shown for low $S N R$ regime and Rayleigh fading. As a benchmark, we also simulate the case where adaptive phase fluctuations are induced in cell 0 but another phase strategy is used in cell $S$ :

- The phase does not vary adaptively in cell $S$ but has a fixed value.

- The phase in cell $S$ varies randomly at each time slot.

- The phase in cell $S$ has a deterministic evolution; it increases by a fixed increment at each time slot (rotates around the trigonometric circle).

- Only thermal noise is considered (cell $S$ does not exist) yielding the simplified expression

$S I N R_{k}^{(0)}(l)=\frac{\left|h_{1 k}^{(0)}(l) \alpha_{1}^{(0)}(l)+h_{2 k}^{(0)}(l) \alpha_{2}^{(0)}(l) e^{j \theta^{(0)}(l)}\right|^{2}}{\frac{2 \sigma^{2}}{\sqrt{10}}}$.

- We replace the thermal noise by additive Gaussian thermal noise; hence,

$$
\operatorname{SINR}_{k}^{(0)}(l)=\frac{\left|h_{1 k}^{(0)}(l) \alpha_{1}^{(0)}(l)+h_{2 k}^{(0)}(l) \alpha_{2}^{(0)}(l) e^{j \theta^{(0)}(l)}\right|^{2}}{2 \sigma^{2}\left(\frac{1}{10}+\frac{1}{\sqrt{10}}\right)}
$$

This is simply obtained by multiplying the performance in absence of interference by the coefficient 0.759 .

In Fig. 7, we further see that increasing the phase by fixed increments in cell $S$ at each time slot does not affect the performance. Thus, keeping a fixed $\theta^{(S)}$ in cell $S$ gives similar results. We observe also that better performances of the scheme are obtained when there is intercell interference noise rather than when the interference noise is replaced by an equal power Gaussian thermal noise.

In Fig. 8, we see that the performance in cell 0 is not affected by the number of users in cell $S$ when adaptive fluctuations are made. The phase strategy in the interfering cell does not seem to affect the performance in cell 0 as a deterministic or a random strategy yields the same results.

\section{INTERFERENCE FROM SiX NEIGHBORING CELlS}

\section{A. Introduction and motivation}

In the previous section, a simplified interference model based on a single fictitious interfering cell and a worstcase approach is introduced. The single interfering cell is first assigned an average interfering power equal to the total interfering power of the six neighboring interfering cells. The approach is further simplified by upper-bounding the average interference noise through a worst-case situation. Specifically, an average attenuation of $10 \mathrm{~dB}$ for the interference noise 


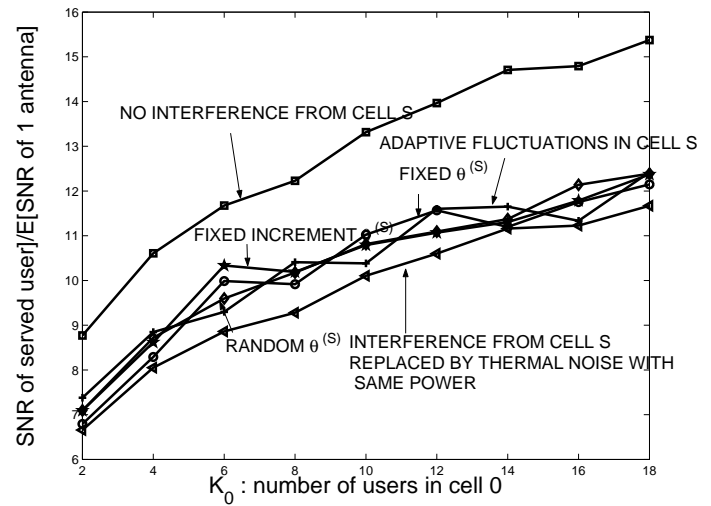

Fig. 7. Performance in cell 0 in presence of interference of cell $S$, low SNR case, Rayleigh fading, Gauss-Markov evolution (slow: $a=0.99$ ), adaptive fluctuations in both cells (two-antennae case), use of various phase strategies in cell $S$.

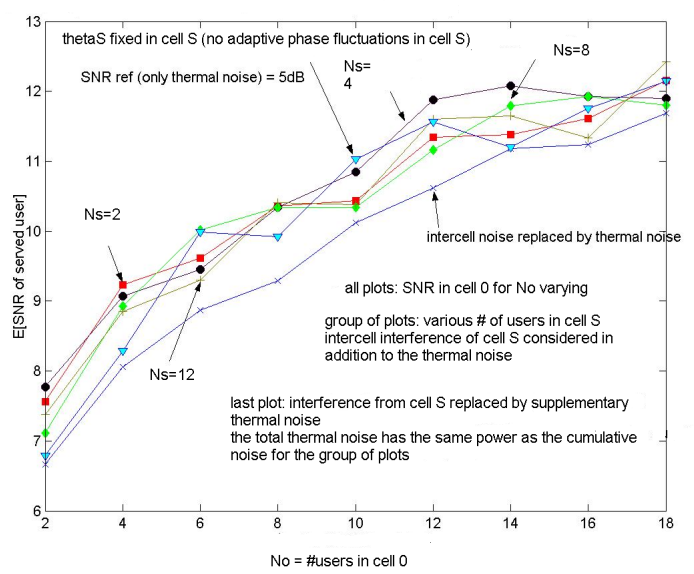

Fig. 8. Performance in cell 0 in presence of interference of cell $S$, low SNR case, Rayleigh fading, Gauss-Markov evolution (slow: $a=0.99$ ), use of adaptive fluctuations in both cells (two-antennae case), various number of users in cell $S$.

is chosen. Consequently, from the total interference-power point of view, cell $S$ represents in a fictitious way the real interference from six cells.

We now present in detail the practical aspects of the general model with six interfering cells introduced at the beginning of this chapter. Phase fluctuations are induced between multiple transmit antennae at any given base station based on the pseudo-random control scheme introduced earlier and using incomplete channel-state-information at the transmitter.

\section{B. Choice of the number of cells in the model}

Here, we would like to consider adaptive phase fluctuations in each cell $i(0 \leq i \leq 6)$ to quantify the impact of six interfering cells over cell 0 . This involves implementing a scheme similar to (17) in each of the seven cells. Although, in principle, the network fans out from cell 0 without limit, our simulation will capture only a finite set $\nu$ of seven cells. Specifically, we introduce for each $i$ such that $0 \leq i \leq 6$ the set $\nu^{(i)}$, which is the first layer of cells interfering with cell

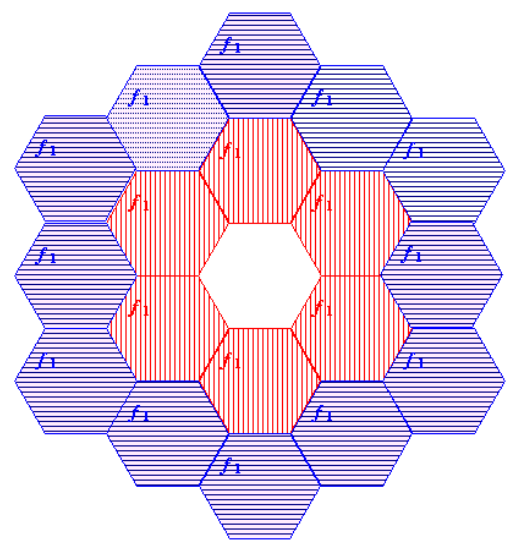

Fig. 9. Layers of cells interfering with cell 0.

$i$. In particular, our study system $\nu$ is $\nu^{(0)} \cup\{$ cello $\}$ and for $i$ such that $0 \leq i \leq 6, \operatorname{card}\left(\nu^{(i)}\right)=6$. We observe that for any $i$ such that $1 \leq i \leq 6$, the set $\nu$ contains only three of the six nodes in set $\nu^{(i)}$. This means that for $1 \leq i \leq 6$, some of the cells interfering with cell $i$ are not part of our study system. Therefore, considering adaptive phase fluctuations in each of the elements of $\nu$ through (17) would involve modeling interactions with cells that are not part of $\nu$.

This situation is illustrated in Fig. 9. For clarity purposes, we have only plotted the cells where the transmission frequency is $f_{1}$. We do have cells with other transmission frequencies so that the frequency reuse factor (equal to $\frac{1}{4}$ ) is respected. The central cell is cell 0 ; the cells with vertical stripes correspond to the first layer of cells interfering with cell 0 , i.e., $\nu^{(0)}$; the cells with horizontal stripes correspond to the additional cells interfering with cells $1-6$, i.e., $\cup_{i=1}^{i=6} \nu^{(i)}$ $-\{$ cell 0$\}$.

To limit our simulations to the cells in $\nu$, we think of two feasible possibilities:

- We consider the model of (17) for cell 0 and open-loop phase strategies for each cell i $(1 \leq i \leq 6)$.

- We consider the model of (17) for cell 0 and we proceed as follows for the interfering terms of cell $i$ where $1 \leq i \leq 6$ : As the interfering noise for a user in cell $i$ is composed of six terms, we multiply by two the interference from the three interfering cells in $\nu^{(0)} \cap \nu^{(i)}$ and choose it as the interfering term. This yields the following formula for user $k$ in cell $i$ where $1 \leq i \leq 6$ :

$$
S I N R_{k}^{(i)}(l)=\frac{\left|U_{k}^{(i)}(l)\right|^{2}}{2 \sum_{j \in \nu^{(0)} \cap \nu^{(i)}} a_{k}^{(0 j)}(l)\left|\widetilde{U}_{k}^{(0 j)}(l)\right|^{2}+\frac{2 \sigma^{2}}{\sqrt{10}}}
$$

\section{Positions of the users in a given cell}

A second issue is the position of the users in the cells of $\nu$. In the simplified model involving a fictitious cell, the positions 


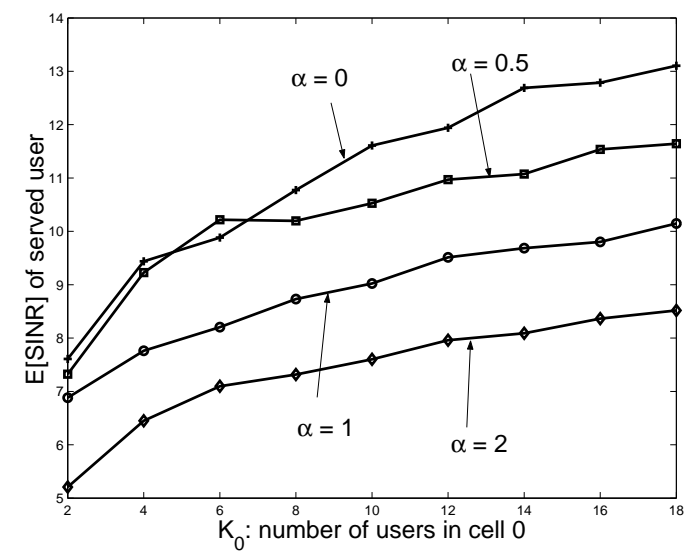

Fig. 10. Performance in cell 0 for six interfering cells, low SNR case, Rayleigh fading, Gauss-Markov evolution (slow: $a=0.99$ ). Use of adaptive fluctuations in cell 0 and open-loop phase strategies in the other cells (twoantennae case).

of the users do not matter because a worst-case model which removes the dependence of the model on the positions of the users is considered. In this section, we improve the precision of the model by considering for each user $k$ in cell $i$ the interfering variable coefficients $a_{k}^{(0 j)}$ for $j \in \nu^{(0)} \cap \nu^{(i)}$ related to its position. We consider uniformly distributing the given number of users over the cell. We can then compute the interfering coefficients for all these positions.

\section{Simulations and observations}

The plots for adaptive phase fluctuations and six interfering cells are presented in Fig. 10. We have considered the case of open-loop phase strategies in the interfering cells (corresponding to the first of two feasible strategies presented previously), Rayleigh fading, and low SNR regime. The plots are done for different values of the parameter $\alpha$. As expected, we observe that the performance worsens when $\alpha$ increases.

\section{CONCLUSION}

In this paper, we presented a scheme using multiple antennae with adaptive phase fluctuations to enhance the downlink of a cellular network. We first showed that the performance could theoretically be improved by adding antennae at the base station and inducing adaptive phase fluctuations between them. However, the feasibility of the scheme has to be taken into account for a practical implementation: there is a tradeoff to achieve between the gain in performance and the practical difficulties that can arise from managing a large number of transmit antennae. We then considered jointly inducing phase and power fluctuations between the transmit antennae. A scheme with only an equal power allocation and adaptive phase fluctuations between the transmit antennae was found in simulations to have better performances than the joint adaptation. We finally considered the impact of the interference of neighboring cells, first through a model based on a single interfering cell, then through a more detailed model based on the interference of six neighboring cells. Analysis and simulations showed that intercell interference damaged the performance less than an equal-power Gaussian thermal noise and that fairness schemes damage the gain of multiuser diversity. Further work could involve:

- Considering other fading models (Ricean fading for example)

- Finding optimal values (for some criterion) of the parameter $\alpha$ introduced in the fairness scheme to achieve a given trade-off between performance and fairness.

- Taking into account the movements of the users in a given cell.

- Building a more consistent model for the impact of the interference of other cells.

To conclude, we can mention the fact that multiuser diversity in wireless networks is currently a topic of much interest. It is already used in the downlink packet scheduling algorithm for Qualcomm's High Data Rate (HDR) system which is optimized for data transfer. It has also been shown recently to greatly increase the throughput of mobile ad-hoc networks for delay tolerant applications [8].

\section{APPENDIX I}

\section{UNIFORM Distribution OF THE POWER ALlOCATION} OVER [0;1] IN THE Single-CELl BEAMFORMING CASE

A complex Gaussian random variable $h_{i k}$ with mean 0 and variance $2 \sigma^{2}$ is equivalent to $h_{i k}=a_{i k}+j b_{i k}$ where $a_{i k}$ and $b_{i k}$ are independent identically distributed: Gaussian with mean 0 and variance $\sigma^{2}$. Under these assumptions, we know that $\sqrt{\left|h_{i k}^{2}\right|}$ has the Rayleigh distribution with parameter $\sigma^{2}$, implying that $\left|h_{i k}^{2}\right|$ has the exponential distribution with parameter $\frac{1}{2 \sigma^{2}}$. Using this fact and the mutual independence of $\left|h_{1 k}^{2}\right|$ and $\left|h_{2 k}^{2}\right|$, we can then easily show that $\alpha_{1}^{2}$ is uniformly distributed over $[0 ; 1]$.

\section{APPENDIX II}

\section{Proof of The Growth RATES For A Single INTERFERING CELL}

First note that $\lambda V_{k}^{(0)}$ and $\lambda \widetilde{V}_{k}^{(S)}$ are independent $\operatorname{Exp}(1)$ random variables.

- Cases 1 and 3: We know that $\max _{1 \leq j \leq n} X_{j}=$ $\sum_{j=1}^{n} \frac{1}{j} X_{j} \quad$ where $\left(X_{j}\right)_{1 \leq j \leq n} \quad$ are independent $\operatorname{Exp}(1)$ random variables. Applying this result to $\left(\lambda V_{k}^{(0)}\right)_{1 \leq k \leq K_{0}}$ in case 1 and taking the expected value, we obtain: $M_{1}=\sqrt{10} \sum_{j=1}^{K_{0}} \frac{1}{j}$. Using the well-known result $\sum_{j=1}^{K_{0}} \frac{1}{j} \sim \log K_{0}$ as $K_{0} \rightarrow+\infty$, the result is shown for case 1 . The result in case 3 is a direct consequence.

- Case 2: Note that for a given $c \geq 0$ :

$$
P\left(\max _{1 \leq k \leq K_{0}} S I N R_{k}^{(0)} \geq c\right)=1-\left(1-\frac{a\left(K_{0}, c\right)}{K_{0}}\right)^{K_{0}}
$$

where $a\left(K_{0}, c\right)=K_{0} P\left(S I N R_{k}^{(0)} \geq c\right)$ and $S I N R_{k}^{(0)}$ is given by (24). Using the fact that $\lambda V_{k}^{(0)}$ and $\lambda \widetilde{V}_{k}^{(S)}$ 
are independent $\operatorname{Exp}(1)$ random variables, we obtain the following:

$$
\begin{aligned}
a\left(K_{0}, c\right) & =K_{0} P\left(\lambda V_{k}^{(0)} \geq c\left(\frac{1}{\sqrt{10}}+\frac{\lambda}{10} \widetilde{V}_{k}^{(S)}\right)\right) \\
& =K_{0} \int_{0}^{+\infty} e^{-c\left(\frac{1}{\sqrt{10}}+\frac{1}{10} v\right)} e^{-v} d v \\
& =e^{-\frac{c}{\sqrt{10}}}\left[\frac{K_{0}}{1+\frac{c}{10}}\right]
\end{aligned}
$$

Let $\epsilon$ denote a real number such that: $0<\epsilon<1$. Using (30), we obtain:

$$
\begin{aligned}
& a\left(K_{0},(1+\epsilon) \sqrt{10} \log K_{0}\right)=\frac{K_{0}{ }^{\epsilon}}{1+\frac{1+\epsilon}{\sqrt{10}} \log K_{0}^{(31)}} \\
& a\left(K_{0},(1-\epsilon) \sqrt{10} \log K_{0}\right)=\frac{K_{0}-\epsilon}{1+\frac{1-\epsilon}{\sqrt{10}} \log K_{0}^{(32)}}
\end{aligned}
$$

Note that $a\left(K_{0},(1+\epsilon) \sqrt{10} \log K_{0}\right) \rightarrow 0 \quad$ and $a\left(K_{0},(1-\epsilon) \sqrt{10} \log K_{0}\right) \rightarrow+\infty$ as $K_{0} \rightarrow+\infty$. Therefore, using (29) and combining it with (31) and (32) respectively, we obtain that $P\left(\max _{1 \leq k \leq K_{0}} S I N R_{k}^{(0)} \geq(1+\epsilon) \sqrt{10} \log K_{0}\right) \rightarrow 0$ and $P\left(\max _{1 \leq k \leq K_{0}} S I N R_{k}^{(0)} \geq(1-\epsilon) \sqrt{10} \log K_{0}\right) \rightarrow$ 1 as $K_{0} \rightarrow+\infty$. Considering $\epsilon \rightarrow 0$ yields the result.

\section{REFERENCES}

[1] R. Knopp and P. Humblet, "Information capacity and power control in single cell multiuser communications," in Proc. IEEE Int. Computer Conf. (ICC'95), vol. 1, Seattle, WA, 1995, pp. 331-335.

[2] D. N. C. Tse, "Multiuser diversity in cellular networks," in Wireless communication seminar slides, Stanford University, Stanford, CA, April 16, 2001.

[3] P. Viswanath, D. N. C. Tse, and R. Laroia, "Opportunistic beamforming using dumb antennas," IEEE Trans. Inform. Theory, vol. 48, pp. 12771294, 2002.

[4] S. Sanghavi, "Adaptive induced fluctuations for multiuser diversity," M. S. thesis, University of Illinois, Urbana-Champaign, IL, 2002.

[5] T. S. Rappaport, Wireless Communications, Principles and Practice. Upper Saddle River, NJ: Prentice-Hall, 2002.

[6] J. Mo and J. Walrand, "Fair end-to-end window-based congestion control," IEEE/ACM Trans. Networking, vol. 8, no. 5, pp. 556-567, 2000.

[7] F. Kelly, "Charging and rate control for elastic traffic," Eur. Trans. Telecommun., vol. 8, pp. 33-37, 2000.

[8] S. N. Diggavi, M. Grossglauser, and D. N. C. Tse, "Even one-dimensional mobility increases ad hoc wireless capacity," in Proc. IEEE International Symposium on Information Theory (ISIT'02), Lausanne, Switzerland, 2002, p. 352. 